\title{
UdS-Sant: English-German Hybrid Machine Translation System
}

\author{
Santanu Pal ${ }^{1}$, Sudip Kumar Naskar ${ }^{2}$, Josef van Genabith ${ }^{\mathbf{1}}$ \\ ${ }^{1}$ Universität des Saarlandes, Saarbrücken, Germany \\ 2 Jadavpur University, Kolkata, India \\ \{santanu.pal, josef.vangenabith\}@uni-saarland.de \\ sudip.naskar@cse.jdvu.ac.in
}

\begin{abstract}
This paper describes the UdS-Sant English-German Hybrid Machine Translation (MT) system submitted to the Translation Task organized in the Workshop on Statistical Machine Translation (WMT) 2015. Our proposed hybrid system brings improvements over the baseline system by incorporating additional knowledge such as extracted bilingual named entities and bilingual phrase pairs induced from example-based methods. The reported final submission is the result of a hybrid system obtained from confusion network based system combination that combines the best performance of each individual system in a multi-engine pipeline.
\end{abstract}

\section{Introduction}

In this paper, we present Universität des Saarlandes (UdS) submission (named UdS-Sant) to WMT 2015 using a Hybrid MT framework. We participated in the generic translation shared task for the English-German (EN-DE) language pair.

Corpus-based MT (CBMT) has delivered progressively improved quality translations since its inception. There are two main approaches to corpus-based MT - Example Based Machine Translation (EBMT) (Carl and Way, 2003) and Statistical Machine Translation (SMT) (Brown et al., 1993; Koehn, 2010). Out of these two, in terms of large-scale evaluations, SMT is the most successful MT paradigm. However, each approach has its own advantages and disadvantages along with its own methods of applying and acquiring translation knowledge from the bilingual parallel training data. EBMT phrases tend to be more linguistically motivated compared to SMT phrases which essentially operate on $n$-grams. The knowledge extraction as well as representation process, in both EBMT and SMT, uses very different techniques in order to extract resources. Even though, SMT is the most popular MT paradigm, it sometimes fails to deliver sufficient quality in translation output for some languages, since each language has its own difficulties.

Multiword Expressions (MWEs) and Named Entities (NEs) offer challenges within a language. MWEs are defined as idiosyncratic interpretations that cross word boundaries (Sag et al., 2002). Named entities on the other hand often consist of more than one word, so that they can be considered as a specific type of MWEs such as noun compounds (Jackendoff, 1997). Traditional approaches to word alignment such as IBM Models (Brown et al., 1993) are unable to tackle NEs and MWEs properly due to their inability to handle many-to-many alignments. In another wellknown word alignment approach, Hidden Markov Model (HMM: (Vogel et al., 1996)), the alignment probabilities depend on the alignment position of the previous word. It does not explicitly consider many-to-many alignment either.

We address this alignment problem indirectly. The objective of the present work is threefold. Firstly, we would like to determine how treatment of MWEs as a single unit affects the overall MT quality (Pal et al., 2010; Pal et al., 2011). Secondly, whether a prior automatic NE aligned parallel corpus as well as example based parallel phrases can bring about any further improvement on top of that. And finally, whether system combination can provide any additional advantage in terms of translation quality and performance.

The remainder of the paper is organised as follows. Section 2 details the components of our system, in particular named entity extraction, translation memory, and EBMT, followed by description of 3 types of Hybrid systems and the system combination module. In Section 3, we outline the complete experimental setup for the shared task and 
provide results and analysis on the performance on the test set in Section 4. Section 5 concludes the proposed research.

\section{System Description}

Our system is designed with three basic components: (i) preprocessing, (ii) hybrid systems and (iii) system combination.

\subsection{Preprocessing}

Data pre-processing plays a very crucial part in any data-driven approach. We carried out preprocessing in two steps:

- Cleaning and clustering sentences based on sentence length.

- Effective preprocessing of data in the form of explicit alignment of bilingual terminology (viz. NEs and MWEs).

The preprocessing has been shown (cf. Section 2.1.2) to improve the output quality of the baseline PB-SMT system (Pal et al., 2013; Tan and Pal, 2014).

\subsubsection{Corpus cleaning}

We utilized all the parallel training data provided by the WMT 2015 shared task organizers for English-German translation. The training data include Europarl, News Commentary and Common Crawl. The provided corpus is noisy and contains some non-German as well as non-English words and sentences. Therefore, we applied a Language Identifier (Shuyo, 2010) on both bilingual English-German parallel data and monolingual German corpora. We discarded those parallel sentences from the bilingual training data which were detected as belonging to some different language by the language identifier. The same method was also applied to the monolingual data.

Successively, the corpus cleaning process was carried out first by calculating the global mean ratio of the number of characters in a source sentence to that in a target sentence and then filtering out sentence pairs that exceed or fall below $20 \%$ of the global ratio (Tan and Pal, 2014). We sorted the entire parallel training corpus based on their sentence length.Tokenisation and punctuation normalisation were performed using Moses scripts. In the final step of cleaning, we filtered the parallel training data on maximum allowable sentence length of 100 and sentence length ratio of 1:2 (either direction). Approximately $36 \%$ sentences were removed from the total training data during the cleaning process.

\subsubsection{Explicit Preprocessing of Terminologies}

Two kinds of terminologies, viz. NEs and MWEs, were considered in the present work. Intuitively, MWEs should be both aligned in the parallel corpus and translated as a whole. However, state-ofthe-art PB-SMT (or any other approaches to SMT) does not generally treat MWEs as special tokens. This is the motivation behind considering MWEs for special treatment in this work. By converting the MWEs into single tokens, we make sure that PB-SMT also treats them as a whole.

NE Alignment (NEA): For NE alignment, we first identify NEs on both sides of the parallel corpus using Stanford NER ${ }^{1}$. Next, we try to align the extracted source and target NEs. If both sides contain only one NE then the alignment is trivial, and we add such NE pairs to seed another parallel NE corpus that contains examples having only one token in both sides. Otherwise, we establish alignments between the source and target NEs using minimum edit distance method. For language pairs having different orthographies (e.g. English-Hindi) NE alignments can be established through transliteration (Pal et al., 2010). If both the source and target sides contain $n$ number of NEs, and the alignments of $n-1$ NEs can be established through minimum edit distance method or by means of already existing alignments, then the $n^{\text {th }}$ alignment is trivial. The bilingual NE pairs extracted thus serve as additional training material and they improve the word alignment at the start of the MT pipeline.

MWE Identification: Translation correspondences between English MWEs and German MWEs are mainly many-to-one correspondences. Therefore, instead of extracting a bilingual MWE list between source and target, we identify the MWEs from the English training sentences and prepare an English MWE list. Once the MWEs are identified, they are converted into single tokens by replacing the spaces with underscores ("_.") so that their alignments can be mapped to single tokens . Before decoding, MWEs in the source side of the testset are also single tokenized by looking up the extracted MWE list. In this experiment, we have followed Point-wise Mutual Infor-

\footnotetext{
${ }^{1}$ http://nlp.stanford.edu/software/CRF-NER.shtml
} 
mation (PMI), Log-likelihood Ratio (LLR), Phicoefficient and Co-occurrence measures for identification of MWEs on the English side. Finally, a system combination model has been developed which provides a normalized score for each of the extracted MWEs. A predefined cut-off score has been considered and the candidates having scores above the threshold value are considered as MWEs.

Example Based Phrase Extraction: We use EBMT techniques to extract additional phrase pairs from the training data to augment the SMT (baseline) phrase pairs in our experiments. We extract EBMT phrase pairs based on the work described in (Cicekli and Güvenir, 2001), a compiled approach of EBMT to automatically extract translation templates from sentence-aligned bilingual text. They observed the similarities and differences between two example pairs. Two types of translation templates, i.e. generalized and atomic templates, are extracted by applying this approach. A generalized translation template replaces similar or differing sequences with variables while an atomic translation template does not contain any variable. The atomic translation templates are used as additional phrase pairs for our Hybrid MT system. This particular approach has a cubic runtime complexity with respect to the number of sentences in the parallel corpus. It takes a significant amount of time to extract phrase pairs even from a small corpus. Therefore we used heuristics to reduce the time complexity. We divided the entire corpus into $n$ clusters based on sentence length such that similar length sentences belong to the same cluster. We extract atomic translations from each of these clusters. For this task, we applied EBMT phrases as addition parallel training example to explicitly enhanced the word alignment model of the MT pipeline.

\subsection{Hybrid System}

The Hybrid approach is investigated by combining multiple knowledge sources such as NEA, EBMT Phrases and MWEs and followed different strategies. As mentioned earlier, we implemented several different systems, namely:

(1) Baseline PB-SMT,

(2) Baseline PB-SMT with NE alignment (NEA),
(3) NEA with EBMT phrase extraction (NEAEBMT),

(4) NEA with EBMT phrase extraction and single-tokenised MWE (NEA-EBMTMWE) and

(5) LM-NEA-EBMT-MWE hybrid system (see Section 2.2.1).

The baseline SMT system is trained on the cleaned English-German parallel corpus. The NEA system makes use of NE aligned parallel data as additional parallel examples. Similarly, EBMT phrase pairs as well as NE aligned data are also used as additional training example in the NEA-EBMT system. The NEA-EBMT-MWE system is very similar to the above mentioned the NEA-EBMT system, the only difference being that the identified source side English MWEs are converted into single tokens for NEA-EBMT-MWE. In order to achieve optimal performance from the component modules, we finally generated a composite translation output using confusion network-based system combination (cf. Section 2.3).

\subsubsection{LM-NEA-EBMT-SMT hybrid system}

In this system, we experiment with the above described models with varying size of monolingual data. We experimented with 4 folds of monolingual data to train the language Models (LM):

- $\mathrm{LM}_{1}$ : Only using the target side (i.e. German) of the parallel training data $(L)$ for language modeling

- $\mathrm{LM}_{2}: L+$ double size of $L$ in terms of number of sentences, collected from the cleaned monolingual corpus

- $\mathrm{LM}_{3}: L+$ triple size of $L$ from the cleaned monolingual corpus

- $\mathrm{LM}_{4}: L+$ all the cleaned monolingual data

Therefore, finally there were 16 different systems (4 systems, i.e., Baseline, NEA, NEAEBMT and NEA-EBMT-MWE, each with 4 LM settings) output available for system combination.

\subsubsection{Post-processing}

As a final step, we try to generate translations of out-of-vocabulary (OOV) words that remain untranslated in the output. These OOV words may 
include some NEs that are already there in the parallel NE list, however they might remain untranslated during decoding. Our system post processed the output by replacing each such OOV NE with the corresponding target language NE after looking up the extracted NE list from the parallel corpus (cf. Section 2.1.2).

\subsection{System Combination}

System Combination is a technique, which combines translation hypotheses (outputs) produced by multiple MT systems. We applied a system combination method on the outputs of the different MT system described earlier. We implement the Minimum Bayes Risk coupled with Confusion Network (MBR-CN) framework described in (Du et al., 2009). The MBR decoder (Kumar and Byrne, 2004) selects the single best hypothesis from amongst the multiple candidate translations by minimising BLEU (Papineni et al., 2002) loss. This single best hypothesis serves as the backbone (also referred to as skeleton) of the confusion network and determines the general word order of the confusion network. A confusion network (Matusov et al., 2006) is built from the backbone while the remaining hypotheses are aligned against the backbone using METEOR (Lavie and Agarwal, 2007) and the TER metric (Snover et al., 2006). The features used to score each arc in the confusion network are word posterior probability, target language model (3-gram, 4-gram), and length penalties. Minimum Error Rate Training (MERT) (Och, 2003) is applied to tune the CN weights (Pal et al., 2014).

\section{Experiment Setup}

\subsection{Baseline Settings}

The effectiveness of the present work is demonstrated by using the standard log-linear PB-SMT model as our baseline system. For building the baseline system, we used a maximum phrase length of 7 and a 5-gram language model. The other experimental settings were: SymGIZA++ aligner (Junczys-Dowmunt and Szał, 2012), which is a modified version of GIZA++ word alignment models by updating the symmetrizing models between chosen iterations of the original word alignment training algorithms and phraseextraction (Koehn et al., 2003). The reordering model was trained on hier-mslr-bidirectional (i.e. using both forward and backward models) and conditioned on both source and target language. The reordering model was built by calculating the probabilities of the phrase pairs being associated with the given orientation such as monotone (m), swap (s) and discontinuous (d). The 5-gram target language model was trained using KENLM (Heafield, 2011). Parameter tuning was carried out using both k-best MIRA (Cherry and Foster, 2012) and Minimum Error Rate Training (MERT) (Och, 2003) on a held-out development set. After the parameters were tuned, decoding was carried out on the held out testset.

Note that all the systems described in Section 2 employ the same PB-SMT settings (apart from the feature weights which are obtained via MERT) as the Baseline system.

\section{Results and Analysis}

As described in Section 2.2.1, we developed 16 different systems. Instead of using all these 16 different systems, we apply only the 6 best performing systems for system combination. Performance is measured on the devset. Table 1 reports the final evaluation results obtained on the test dataset. The best 6 systems are as follows:

- System 1: NEA-EBMT (selective high frequency phrases) with baseline PB-SMT settings and $\mathrm{LM}_{1}$.

- System 2: System 1 experimental settings + single tokenised source MWEs (i.e. NEAEBMT-MWE, cf. Section 2.2).

- System 3: System 2 with MIRA-MERT coupled tuning.

- System 4: System 3 with $\mathrm{LM}_{2}$.

- System 5: System 3 with $\mathrm{LM}_{3}$.

- System 6: System 3 with $\mathrm{LM}_{4}$.

System 6 provides the individual best system. System combination (System-7 in Table 1) of the 6 best performing individual systems brings considerable improvements over each of the individual component systems.

\section{Conclusions and Future Work}

A hybrid system (System 6) with NE alignment, EBMT phrases, single-tokenized source MWEs, and MIRA-MERT coupled tuning results in the best performing system. However, confusion 


\begin{tabular}{lccc}
\hline Systems & BLEU & BLEU(Cased) & TER \\
\hline Baseline & 16.7 & 16.2 & 89.6 \\
System 1 & 18.1 & 17.5 & 88.2 \\
System 2 & 18.1 & 17.6 & 87.8 \\
System 3 & 19.0 & 18.4 & 85.3 \\
System 4 & 20.0 & 19.5 & 84.1 \\
System 5 & 20.3 & 19.7 & 83.8 \\
System 6 & 20.7 & 20.2 & 83.5 \\
System 7 & $\mathbf{2 2 . 6}$ & $\mathbf{2 2 . 1}$ & $\mathbf{8 2 . 3}$ \\
\hline
\end{tabular}

Table 1: Results.

network-based system combination outperforms all the individual MT systems. The fact that the systems were tuned with BLEU scores may be one of the reasons behind the poor TER scores produced by the systems. In future, we will carry out in depth investigation of the impacts of MWEs within the current experimental settings. We will also analyze the usability and contribution of the novel EBMT phrases in the SMT decoder.

\section{Acknowledgments}

The research leading to these results has received funding from the EU FP7 Project EXPERT - the People Programme (Marie Curie Actions) (Grant No. 317471)

\section{References}

Peter F Brown, Vincent J Della Pietra, Stephen A Della Pietra, and Robert L Mercer. 1993. The Mathematics of Statistical Machine Translation: Parameter Estimation. Computational linguistics, 19(2):263311.

Michael Carl and Andy Way. 2003. Recent advances in example-based machine translation, volume 21. Springer Science \& Business Media.

Colin Cherry and George Foster. 2012. Batch Tuning Strategies for Statistical Machine Translation. In Proceedings of the 2012 Conference of the North American Chapter of the Association for Computational Linguistics: Human Language Technologies, pages $427-436$.

Ilyas Cicekli and H Altay Güvenir. 2001. Learning Translation Templates From Bilingual Translation Examples. Applied Intelligence, 15(1):57-76.

Jinhua Du, Yifan He, Sergio Penkale, and Andy Way. 2009. MATREX: The DCU MT System for WMT 2009. In Proceedings of the 4th Workshop on Statistical Machine Translation, pages 95-99, March.
Kenneth Heafield. 2011. KenLM: Faster and Smaller Language Model Queries. In Proceedings of the Sixth Workshop on Statistical Machine Translation, pages 187-197.

Ray Jackendoff. 1997. The architecture of the language faculty. Number 28. MIT Press.

Marcin Junczys-Dowmunt and Arkadiusz Szał. 2012. SyMGiza++: Symmetrized Word Alignment Models for Statistical Machine Translation. In Proceedings of the 2011 International Conference on Security and Intelligent Information Systems, pages 379390.

Philipp Koehn, Franz Josef Och, and Daniel Marcu. 2003. Statistical Phrase-based Translation. In Proceedings of the 2003 Conference of the North American Chapter of the Association for Computational Linguistics on Human Language Technology, pages 48-54.

Philipp Koehn. 2010. Statistical Machine Translation. Cambridge University Press, New York, NY, USA, 1st edition.

Shankar Kumar and William Byrne. 2004. Minimum Bayes Risk Decoding for Statistical Machine Translation. In Proceedings of the North American Association for Computational Linguistics (NAACL), pages 169-176, March.

Alon Lavie and Abhaya Agarwal. 2007. METEOR: An Automatic Metric for MT Evaluation with High Levels of Correlation with Human Judgments. In Proceedings of the Second Workshop on Statistical Machine Translation, pages 228-231.

Evgeny Matusov, Nicola Ueffing, and Hermann Ney. 2006. Computing Consensus Translation from Multiple Machine Translation Systems Using Enhanced Hypotheses Alignment. In Conference of the European Chapter of the Association for Computational Linguistics, pages 33-40, Trento, Italy, April.

Franz Josef Och. 2003. Minimum Error Rate Training in Statistical Machine Translation. In Proceedings of the 41st Annual Meeting on Association for Computational Linguistics - Volume 1, pages 160-167.

Santanu Pal, Sudip Kumar Naskar, Pavel Pecina, Sivaji Bandyopadhyay, and Andy Way. 2010. Handling Named Entities and Compound Verbs in PhraseBased Statistical Machine Translation. In In Proceedings of the of Multiword Expression Workshop (MWE-2010). The 23rd International conference of computational linguistics (Coling 2010).

Santanu Pal, Tanmoy Chakraborty, and Sivaji Bandyopadhyay. 2011. Handling Multiword Expressions in Phrase-Based Statistical Machine Translation. Machine Translation Summit XIII, pages 215224. 
Santanu Pal, Sudip Kumar Naskar, and Sivaji Bandyopadhyay. 2013. MWE Alignment in Phrase Based Statistical Machine Translation. The XIV Machine Translation Summit, pages 61-68.

Santanu Pal, Ankit Srivastava, Sandipan Dandapat, Josef van Genabith, Qun Liu, and Andy Way. 2014. USAAR-DCU Hybrid Machine Translation System for ICON 2014. In Proceedings of the 11th International Conference on Natural Language Processing, Goa, India.

Kishore Papineni, Salim Roukos, Todd Ward, and WeiJing Zhu. 2002. BLEU: A Method for Automatic Evaluation of Machine Translation. In Proceedings of the 40th Annual Meeting on Association for Computational Linguistics, ACL '02, pages 311-318.

Ivan A Sag, Timothy Baldwin, Francis Bond, Ann Copestake, and Dan Flickinger. 2002. Multiword Expressions: A Pain in the Neck for NLP. In Computational Linguistics and Intelligent Text Processing, pages 1-15. Springer.

Nakatani Shuyo. 2010. Language Detection Library for Java.

Matthew Snover, Bonnie Dorr, Richard Schwartz, Linnea Micciulla, and John Makhoul. 2006. A study of Translation Edit Rate with Targeted Human Annotation. In Proceedings of association for machine translation in the Americas, pages 223-231.

Liling Tan and Santanu Pal. 2014. Manawi: Using Multi-word Expressions and Named Entities to Improve Machine Translation. In Proceedings of Ninth Workshop on Statistical Machine Translation.

Stephan Vogel, Hermann Ney, and Christoph Tillmann. 1996. HMM-based word alignment in statistical translation. In Proceedings of the 16th conference on Computational linguistics-Volume 2, pages 836841. Association for Computational Linguistics. 